Published in final edited form as:

Nat Genet. 2013 September ; 45(9): 964-965. doi:10.1038/ng.2736.

\title{
APOBEC3B mutagenesis in cancer
}

\author{
Kawai J Kuong and Lawrence A Loeb \\ Department of Pathology and Biochemistry, University of Washington, Seattle, Washington, USA. \\ laloeb@u.washington.edu
}

\begin{abstract}
Recent evidence has implicated $A P O B E C 3 B$ as a source of mutations in cervical, bladder, lung, head and neck, and breast cancers. APOBEC enzymes normally function in innate immune responses, including those that target retroviruses, suggesting links between mutagenesis, immunity and viral infection in the process of cancer development.
\end{abstract}

Human cancer genomes are exceptionally complex, as they contain tens of thousands of mutations. Despite this complexity, the observed nucleotide substitutions may provide fingerprints to indict specific mechanisms in carcinogenesis. In this issue of Nature Genetics, two papers, one from Reuben Harris and colleagues ${ }^{1}$ and a second from Dmitry Gordenin, Gad Getz and colleagues ${ }^{2}$, demonstrate that a gene encoding one member of the APOBEC (apolipoprotein B mRNA editing enzyme, catalytic polypeptide-like) family of cytidine deaminases, $A P O B E C 3 B$, leaves a tell-tale mutation signature that is enriched in the genomes of many human cancers. APOBEC family members normally function as part of the innate immune system that protects against retrovirus and retrotransposon propagation, such as restricting HIV-1 viral reverse transcription ${ }^{3}$. However, these enzymes can also deaminate cytosines in the host genome and generate $\mathrm{C} \rightarrow \mathrm{T}$ mutations ${ }^{4-6}$.

In a previous study, Burns et al. ${ }^{4}$ provided evidence that APOBEC $3 \mathrm{~B}$ is overexpressed in breast tumors and cell lines and that the $A P O B E C 3 B$ mutation signature is statistically more prevalent in the breast tumor database of The Cancer Genome Atlas (TCGA) than is expected ${ }^{4}$. In this issue, Burns et al. ${ }^{1}$ extend their study to include 19 different human tumors. They systematically analyzed mutation frequencies, spectra and sequence contexts using whole-exome mutation data from TCGA. Their RNA sequencing (RNA-seq) studies indicated that APOBEC 3B is overexpressed in several human cancer types, and this overexpression correlates with the presence of the $A P O B E C 3 B$ mutation signature. These analyses, together with evidence from previous studies ${ }^{4,6,7}$, suggest that $A P O B E C 3 B$ may contribute to the cytosine mutation clusters observed in many cancers ${ }^{5,6}$.

The paper by Roberts et al. ${ }^{2}$ provides an in-depth analysis of both whole-genome and whole-exome sequencing data sets from various sources, including TCGA. Using wholegenome data sets, the authors found that many mutations are clustered and localized at breakpoints of chromosomal rearrangements, which is consistent with the known specificity of APOBEC family members ${ }^{3,6}$ for single-stranded DNA. Interestingly, the APOBEC $3 B$ mutation signature was detectable in colorectal and prostate cancers only when wholegenome, but not whole-exome, data were used, suggesting a tissue-specific bias against enrichment of mutations by $A P O B E C 3 B$ in coding regions. In addition, their analysis

(C) 2013 Nature America, Inc. All rights reserved.

COMPETING FINANCIAL INTERESTS

The authors declare no competing financial interests. 
showed that the HER2-enriched subtype of breast cancer harbors an exceptionally high frequency of $A P O B E C 3 B$-associated mutations as compared with other breast cancer subtypes. Both studies from Burns et al. ${ }^{1}$ and Roberts et al. ${ }^{2}$ independently reached the same conclusion that the $A P O B E C 3 B$ mutation signature is specifically enriched in six types of cancers, including those of the cervix, bladder, lung (adeno and squamous cell), head and neck, and breast.

\section{APOBEC3B mutation signature}

Mutation signatures have aided in the identification of environmental mutagens and carcinogens (Fig. 1) ${ }^{7,8}$. $\mathrm{C} \rightarrow \mathrm{T}$ transitions in cervix, bladder, lung, head and neck, and breast cancers have been suggested to be caused by APOBEC3B on the basis of the positive association of the distinct mutation signature and overexpression of APOBEC $3 \mathrm{~B}^{1,2}$. Cytosine deamination by APOBEC3B results in a uracil residue. Excision of the uracil residue by uracil DNA glycosylase generates an apurinic (AP) site, and insertion of adenine opposite the AP site results in $\mathrm{C} \rightarrow \mathrm{T}$ transitions ${ }^{9}$. Alternatively, DNA polymerases can replicate across the uracil residue directly and insert an adenine, which also results in $\mathrm{C} \rightarrow \mathrm{T}$ transitions ${ }^{9}$. AP sites and $\mathrm{C} \rightarrow \mathrm{T}$ mutations can also arise by other processes (Fig. 1), including spontaneous or chemical-induced cytosine deamination, error-prone bypass after ultraviolet light damage or DNA polymerase errors ${ }^{7,9}$. The contribution of other mutagenic processes in the cancers that are associated with APOBEC3B mutagenesis is still unclear. One can partially discriminate between the various events leading to cytosine substitutions using the sequence of the adjacent nucleotides. APOBEC3B preferentially deaminates cytosine residues when it is adjacent to a $5^{\prime}$ thymine $6,10,11$ and a $3^{\prime}$ thymine or adenine ${ }^{4}$. In the current studies, only cytosine substitutions that occur within the trinucleotide TㅁA or TㄷT sequence context are attributed to APOBEC3B mutagenesis ${ }^{1,2}$. However, the contribution of other mutagens to cytosine substitutions in these sequence contexts cannot be ruled out. Further studies are also warranted to identify the translesion polymerase that contributes to the $\mathrm{C} \rightarrow \mathrm{G}$ and $\mathrm{C} \rightarrow \mathrm{A}$ mutations observed at these sequence motifs ${ }^{1,2}$.

\section{Immunity, mutagenesis and cancer}

Other APOBEC enzymes could also act as mutators. Overexpression of two other cytidine deaminases has been shown to cause cancer in transgenic mice: overexpression of Apobec1 causes hepatocellular carcinoma ${ }^{12}$, whereas overexpression of activation-induced deaminase (Aid) causes $\mathrm{T}$ cell lymphomas ${ }^{13}$. Overexpression of Apobec $3 \mathrm{~b}$ in animal models may confirm its tumor-type specificity.

Elucidation of the signals that lead to overexpression of APOBEC family members in cancer cells may reveal how viral infection and immune responses are associated with carcinogenesis. Expression of AID and mutagenesis have been shown to be induced by Helicobacter pylori infections in normal gastric epithelia ${ }^{14}$. Intriguingly, both the current studies $^{1,2}$ and recent work from Lawrence et al..$^{7}$ separately showed that the APOBEC $3 B$ mutation signature is enriched in cervical and head and neck cancers, and a major risk factor for the development of these cancers is infection by human papilloma virus. It will be interesting to determine whether viral infections can trigger APOBEC $3 \mathrm{~B}$ mutagenesis and whether variation in infection and immune status can explain why APOBEC $3 \mathrm{~B}$ is associated with cancers in some tissues but not others.

On the basis of these studies, it is reasonable to hypothesize that inhibitors of APOBEC3B may prevent mutation accumulation in specific human cancers. Alternatively, it is postulated that APOBEC-mediated mutagenesis of viral DNA may result in an increase in viral mutation load to a level that exceeds the threshold for viral viability ${ }^{3,10}$. Accordingly, 
induction of APOBEC family members in certain human tumors with an existing high mutation load may similarly increase mutation numbers to a point that exceeds tumor viability ${ }^{15}$.

\section{References}

1. Burns MB, Temiz NA, Harris RS. Nat. Genet. 2013; 45:977-983. [PubMed: 23852168]

2. Roberts SA, Getz G, Gordenin DA. Nat. Genet. 2013; 45:970-976. [PubMed: 23852170]

3. Harris RS, Liddament MT. Nat. Rev. Immunol. 2004; 4:868-877. [PubMed: 15516966]

4. Burns MB, et al. Nature. 2013; 494:366-370. [PubMed: 23389445]

5. Lada AG, et al. Biol. Direct. 2012; 7:47. [PubMed: 23249472]

6. Taylor BJ, et al. eLife. 2013; 2:e00534. [PubMed: 23599896]

7. Lawrence MS, et al. Nature. 2013; 499:214-218. [PubMed: 23770567]

8. Nik-Zainal S, et al. Cell. 2012; 149:979-993. [PubMed: 22608084]

9. Loeb LA, Preston BD. Annu. Rev. Genet. 1986; 20:201-230. [PubMed: 3545059]

10. Bishop KN, et al. Curr. Biol. 2004; 14:1392-1396. [PubMed: 15296758]

11. Beale RC, et al. J. Mol. Biol. 2004; 337:585-596. [PubMed: 15019779]

12. Yamanaka S, et al. Proc. Natl. Acad. Sci. USA. 1995; 92:8483-8487. [PubMed: 7667315]

13. Robbiani DF, Nussenzweig MC. Annu. Rev. Pathol. 2013; 8:79-103. [PubMed: 22974238]

14. Matsumoto Y, et al. Nat. Med. 2007; 13:470-476. [PubMed: 17401375]

15. Prindle MJ, Fox EJ, Loeb LA. Curr. Drug Targets. 2010; 10:1296-1303. [PubMed: 20840072] 


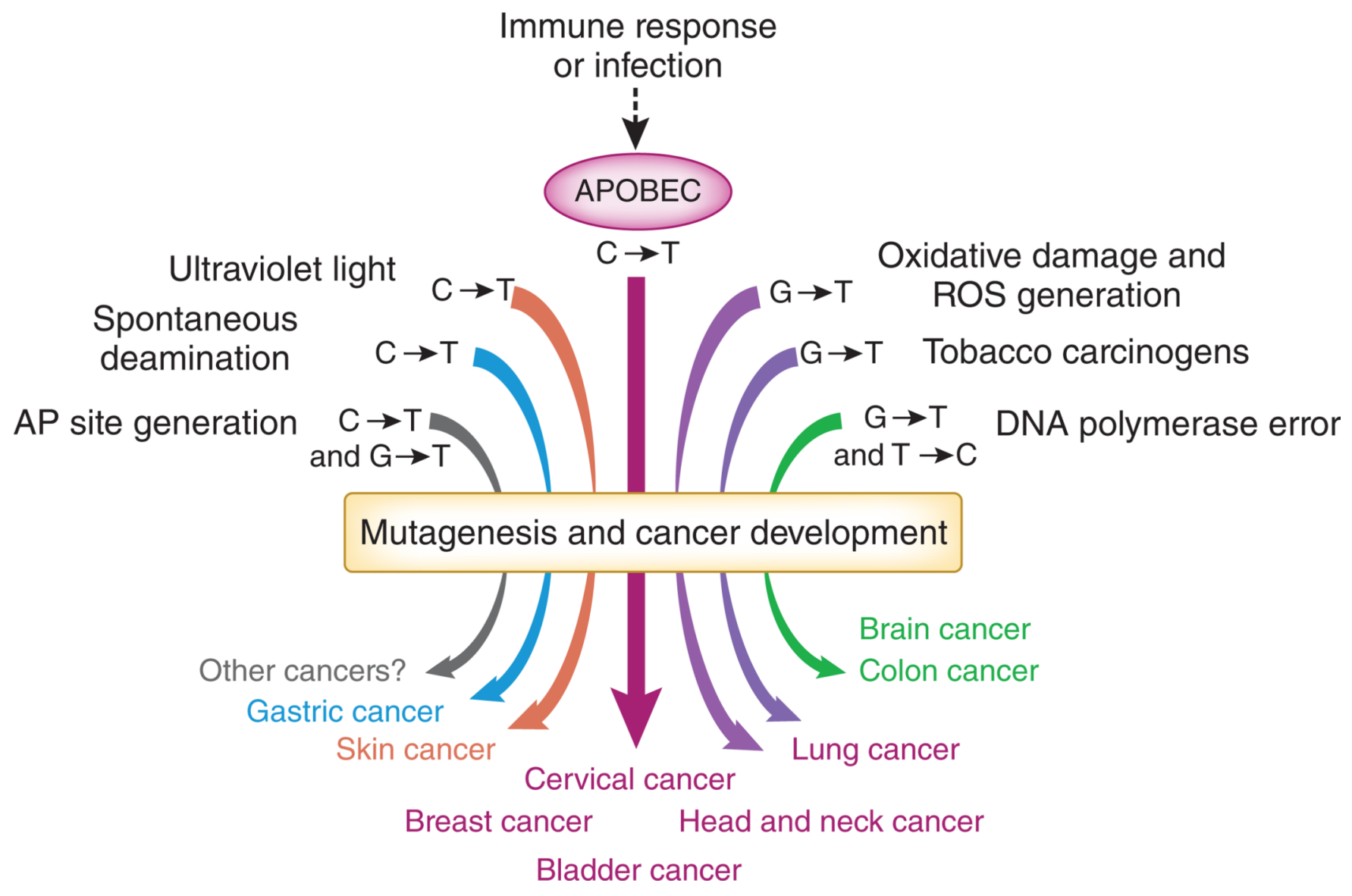

Figure 1.

Mutation signatures and cancer. There are many sources of mutagenic activity that contribute to tumorigenesis. $A P O B E C 3 B$, which has a known role in innate immune responses, is now implicated as a source of mutation in cervical, bladder, lung, head and neck, and breast cancers. ROS, reactive oxygen species. 